\title{
Wearable System for Daily Activity Recognition Using Inertial and Pressure Sensors of a Smart Band and Smart Shoes
}

\author{
P.H. Truong, S. You, S.-H. Ji, G.-M. Jeong
}

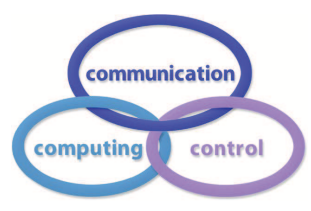

Phuc Huu Truong

Korean Institute of Industrial Technology, Korea

phtruong@kitech.re.kr

\section{Sujeong You}

Korean Institute of Industrial Technology, Korea

sjyou21@kitech.re.kr

\section{Sang-Hoon Ji}

Korean Institute of Industrial Technology, Korea

robot91@kitech.re.kr

\section{Gu-Min Jeong*}

School of Electrical Engineering, Kookmin University, Jeongneung-dong, Seongbuk-gu, 02707 Korea

*Corresponding author: gm1004@kookmin.ac.kr

\begin{abstract}
Human Activity Recognition (HAR) is a challenging task in the field of human-related signal processing. Owing to the development of wearable sensing technology, an emerging research approach in HAR is to identify user-performed tasks by using data collected from wearable sensors. In this paper, we propose a novel system for monitoring and recognizing daily living activities using an off-the-shelf smart band and two smart shoes. The system aims at providing a useful tool for solving problems regarding body part placement, fusion of multimodal sensors and feature selection for a specific set of activities. The system collects inertial and plantar pressure data at wrist and foot to analyze and then, extract, select important features for recognition. We construct and compare two predictive models of classifying activities from the reduced feature set. A comparison of the classification for each wearable device and a fusion scheme is provided to identify the best body part for activity recognition: either the wrist or the feet. This comparison also demonstrated the effective HAR performance of the proposed system.

Keywords: Human Activity Recognition (HAR); Daily Activity Recognition (DAR); Daily Living Activity (DLA); Feature Selection; Smart-Band; Smart-Shoes.
\end{abstract}

\section{Introduction}

Human Activity Recognition (HAR) is an emerging research area in human-machine interaction $[5,16,19]$. HAR is also an essential aspect of other fields, especially localization $[21,26]$, health monitoring [28], medical diagnostics and rehabilitation [14,33]. HAR has challenges that are common with those in other recognition areas, such as face recognition and speech recognition. Some of these challenges include the determination of which attributes to measure, the design of feature-extraction algorithms, and the development of efficient computational techniques. Nevertheless, HAR exhibits higher complexity because human activities are more various to formulate 
its structure compared with face or speech. Additionally, HAR requires different sensing systems for different applications. The recognition of some certain periodic activities, such as walking, is clear $[20,37]$. However, the recognition of other activities is challenged because people performs a lot of activities a day. Moreover, people occasionally perform many activities at the same time.

Recently, the development of sensor technologies, especially wearable sensors, has advanced numerous design for activity recognition $[4,8,18,22]$. Wang et al. [35] used a single waist-worn triaxial accelerometer to recognize six activities based on a hidden Markov model. Banos et al. [2] presented a mobile system based on wearable inertial and electromyography sensors to measure trunk postures and muscle signals. Their evaluation proved that the system can be acceptable for use in measuring trunk endurance. Laudanski et al. [17] mounted two inertial measurement units (IMU) consisting of accelerometer and gyroscope sensors onto user's shanks to classify gait activities. After deriving frequency features, a k-nearest neighbor (KNN) algorithm was used to classify the activities. Najafi et al. [23] developed an ambulatory system using accelerometer and gyroscope sensors, and attached it onto human chest to recognize daily activities. Storm et al. [32] utilized commercial instruments to examine step detection and HAR using triaxial accelerometers. In their study, HAR was performed using a DynaPort move-monitor worn on the lower back and an ActivPAL kit attached to the shanks of users. Their method performed well in terms of recognizing five basic ambulatory activities.

HAR systems based on smartphones have also been developed [26, 27, 29,30]. Pei et al. [26] constructed a Least Square SVM-based model to classify six activities using sensors from a smartphone. Using the smartphone's inertial data, a HAR dataset was created and published as a benchmark for human activity recognition [27]. On this database, San-Segundo et al. [30] created frequency features and developed a Gaussian Mixture model to segment the activities. Another approach used for HAR systems is based on smart watches [11,24,29]. Gyorbiro et al. [11] used a smart watch connected to a smartphone via Bluetooth to collect acceleration, magnetic, and angular speed data of six activities at the wrist, hip, and ankle. A neural network model was then applied to classify these activities. Moreover, convolution neural network (CNN) have been previously applied to recognize human activities using inertial data from smartphones [29].

HAR systems based on smart shoes have also been presented $[13,38]$. In a previous publication, we used two smart shoes to classify three ambulatory activities based on a new pressurebased feature. Zhang et al. utilized foot-force sensors and derived basic statistical features to classify five mobile activities. Fusing the aforementioned sensing approaches, different models have been presented to solve the HAR problem [3,9,20]. Bao et al. [3] classified twenty activities using five biaxial accelerometers on the arm, hip, thigh, wrist, and ankle with an accuracy of $84.26 \%$ using decision tree classifiers. Huynh et al. [12] placed totally 12 accelerometers on the shoulders, wrists, elbows, knees, ankles and both sides of the hip and then combined multiple eigenspaces with Support Vector Machine (SVM) to recognize eight daily activities. In summary, in the approaches focused on wearable sensors, researchers commonly addressed HAR by identifying tasks performed by users using inertial sensors, particularly accelerometers.

Many features derived from acceleration data have been presented for addressing HAR problems. These features are essentially based on statistical computations such as the mean, median, $[1,26,34,36]$, or correlation between the axes of acceleration $[6,10,36]$. Features have also been created based on signal-magnitude processing [6,11]. Another category of accelerationderived features is frequency-based features $[3,7]$. Several researchers have summarized the presented features and tested them with particular sensor setups $[1,10]$. Gonzalez et al. [10] used two smart watches to record accelerations at the wrists of users during three activities, namely walking, standing and resting, and then derived a list of common statistical features. A filter feature selection (FS) and a wrapper feature selection were then applied sequentially to select important feature sets for classification. A Genetic Fuzzy Finite State Machine (GFFSM) model 
was presented to classify activities based on the selected feature sets.

Although advances in wearable sensors have facilitated the design and deployment of HAR systems, developing HAR applications with high accuracy remains a challenging task. It is because human activity is highly diverse, and therefore, HAR requires effective selection and efficient incorporation of sensors to obtain an accurate classification. In this paper, we aim at addressing some of the important issues in wearable-sensors-based HAR regarding sensor placement, sensor fusion, and feature selection from inertial and plantar-based data. These issues are evaluated in a user-dependent approach for daily activity recognition using a smart band and two smart shoes. We design a wearable monitoring system using a smart band, a pair of smart shoes and a smartphone to synchronously gather data from two users. The wearable monitoring system gathers inertial data at the user's wrist using built-in accelerometer and gyroscope sensors of the smart band. Using the sensors integrated into the smart shoes, the system collects acceleration, angular speed, and plantar pressure from the left and right feet. The plantar pressure consists of eight sensing data from eight metatarsal areas on each foot. The collected data are used to analyze and recognize the daily activities. The contribution of the paper includes: presenting a wearable monitoring system that can synchronously track human activities; exploring the optimal body part between the wrist and feet to attach sensors for the best HAR; evaluating activity classification using a data-fusion platform; examining state-of-the art features derived from inertial data to determine the useful features for activities; comparing predictive models for classifying daily activities.

The remainder of this paper is organized as follows. Section 2 provides the details of the wearable monitoring system that is used to collect data from the daily activities. Section 3 describes the nine aforementioned activities, summarizes the state-of-the-art features, and explains the feature-selection method and the predictive models for recognizing these activities. The experimental results are presented in Section 4. Finally, we conclude and identify future works in Section 5 .

\section{Wearable sensing system for daily activity monitoring}

In this section, we describe the design of the proposed wearable sensing system and the techniques used to synchronously collect inertial and plantar-pressure data from the wearable devices. To recognize human activities, we created a wearable sensing system to acquire the motion data of user activities based on a smart band and two smart shoes. We used the smart band to obtain triaxial acceleration and triaxial angular speed data from the participants' wrist, whereas, the smart shoes were utilized to collect triaxial acceleration and eight pressure values from the insoles of shoes. These devices were connected to a smartphone via Bluetooth to control and synchronize data acquisition. Figure 1 shows the architecture of the proposed sensing system.

Gao et al. [9] stated that recognition accuracy does not vary more than $1 \%$ when the sampling rate is increased above $50 \mathrm{~Hz}$. In [31], authors concluded that using a low sampling rate $(25 \mathrm{~Hz})$ for accelerometers and pressure sensors possibly produces high accuracy for posture and activity recognition. Therefore, to save energy and facilitate implementation, we set the sampling rate of the wearable system to $50 \mathrm{~Hz}$. We required the user to wear the smart shoes and smart band and to hold the smartphone in his pocket during experimentation. The participants conducted each separate activity for a specific duration. The collected data are continuously saved into a SQL database that can be uploaded to cloud for remote processing. The database is also used for offline analysis and classification to evaluate the performance in terms of the mentioned issues. 


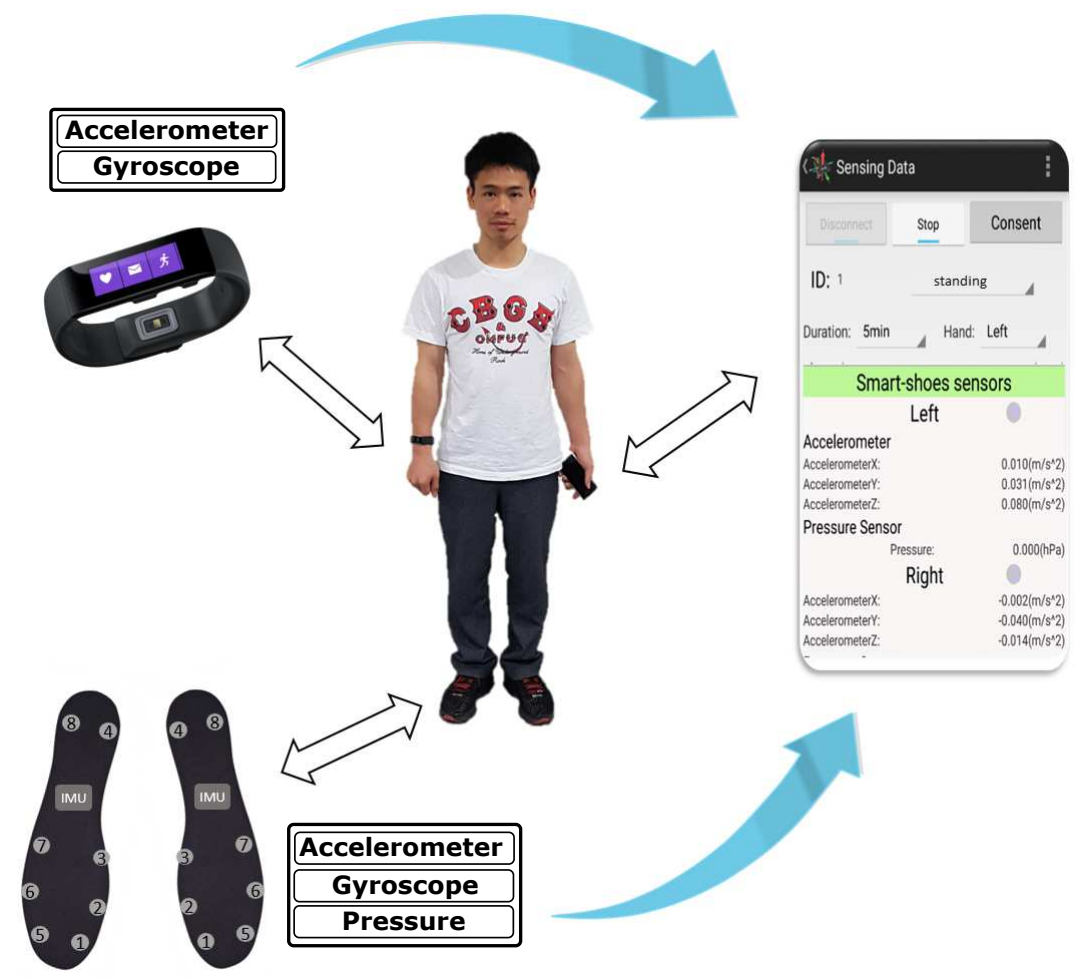

Figure 1: Wearable sensing system

\section{Daily activity recognition}

This section describes the activities of interest and the experimental setup in the study. We depict and analyze the corresponding inertial and plantar-pressure data of these activities obtained from the right wrist and both feet. In particular, we consider nine daily activities that require movement of both hands and feet. Figure 2 shows the activities studied in the paper.

The nine conducted activities were formed from three groups of similar activities, as described in Table 1. Group 1 represents ambulatory activities, which are closely related to foot movement. Group 2 represents activities that are dominated by hand movement. Finally, group 3 represents activities that include a balanced mixture of hand and foot movement. The combination of these activity groups is used to evaluate the importance of the relationship between the sensor placement and the activities.

Table 1: Groups of daily activities.

\begin{tabular}{|c|c|c|c|}
\hline Group & \multicolumn{3}{|c|}{ Activity (Denotation) } \\
\hline 1 & Standing (A1) & Walking (A2) & Jogging (A3) \\
\hline 2 & Window cleaning (A4) & Tooth brushing (A5) & PC using (A6) \\
\hline 3 & Door opening (A7) & Floor mopping (A8) & Book carrying (A9) \\
\hline
\end{tabular}

To recognize activities, the sensory data from different sensors in the three devices are first smoothed using a low-pass Butterworth filter. The filtered data are then merged sequentially to evaluate their importance in activity recognition. Then, we derive all possible features from these data based on statistics, customization, and energy computation. Finally, we analyze and rank all of the features derived from the sensory data, and select subsets of important features for classification. This process is described in Figure 3. 


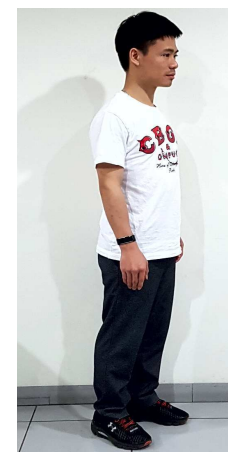

(a)

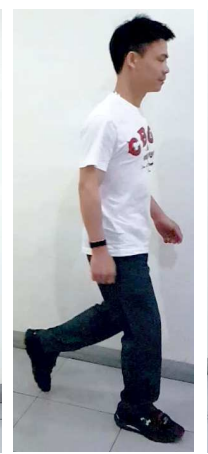

(b)

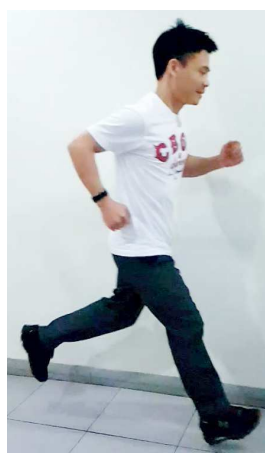

(c)

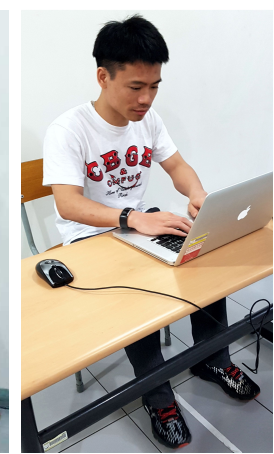

(d)

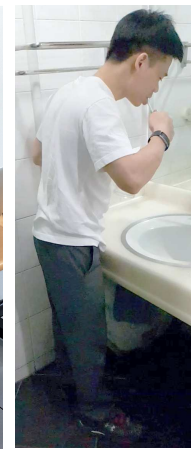

(e)

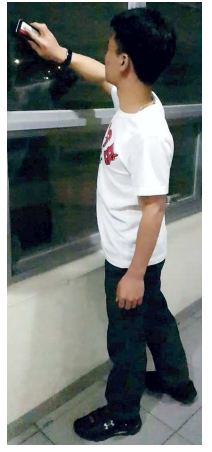

(f)

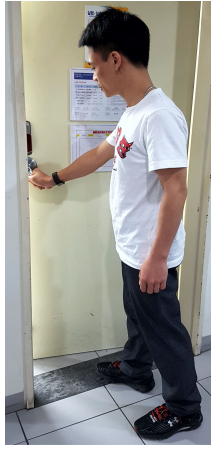

(g)

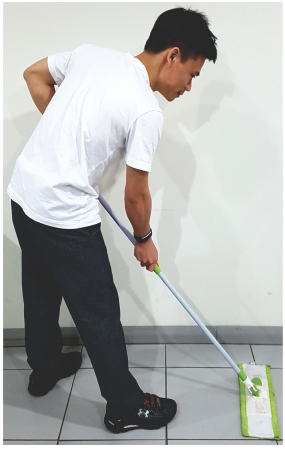

(h)

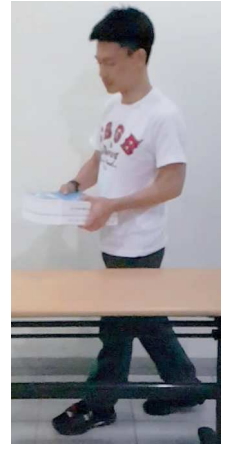

(i)

Figure 2: Descriptions of the experimental activities: (a) Standing; (b) Walking; (c) Jogging; (d) PC Using; (e) Tooth Brushing; (f) Window Cleaning; (g) Door Opening; (h) Floor Mopping; (i) Book Carrying

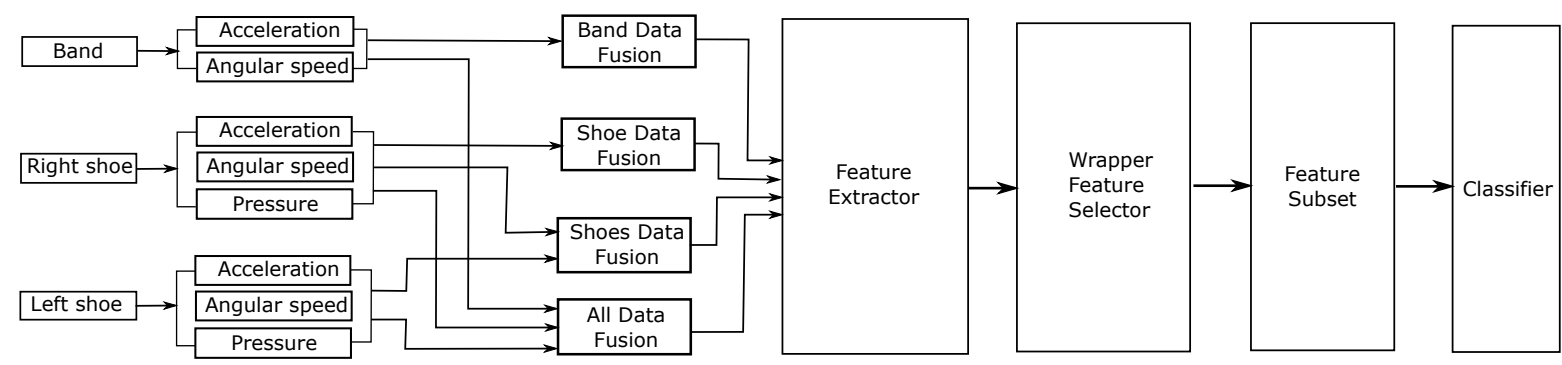

Figure 3: Architecture of activity recognition

\subsection{Experimental configuration}

Users wear the sensor devices and run the wearable system while conducting the activities. The wearable sensing system continuously records all sensory data and saves data in a SQLformatted dictionary for offline processing. Figure 4 and 5 depict the acceleration and angular speed, respectively, obtained at a user's wrist for the nine activities. The angular speed data of the activities on the right shoe are shown in Figure 6.

Herein, we accounted for the issues in offline-segmented activity recognition, by starting the system immediately before the activities begans and stopping it immediately after the activities concluded, which ensured that only the motion data from the activities were obtained. Then, in the offline database, we divided the data into equal-time segments for all activities. We used both the event-defined and the sliding windows for segmentation. We allowed the users 


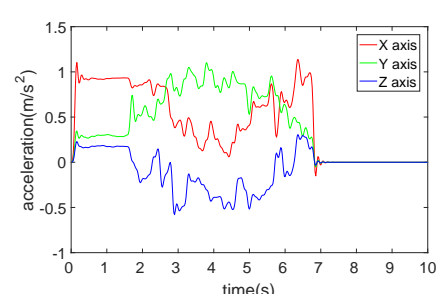

(a)

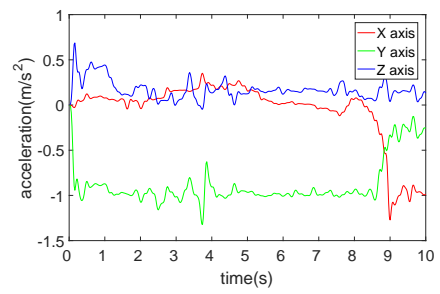

(d)

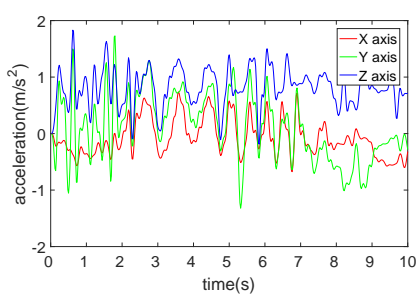

(g)

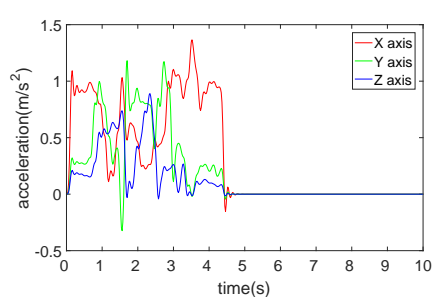

(b)

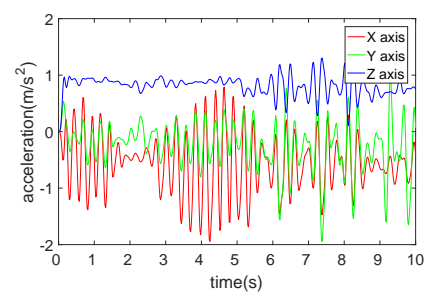

(e)

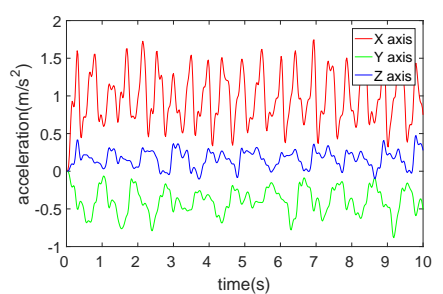

(h)

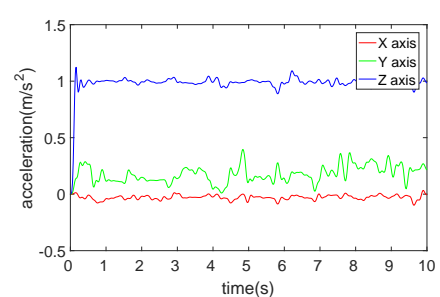

(c)

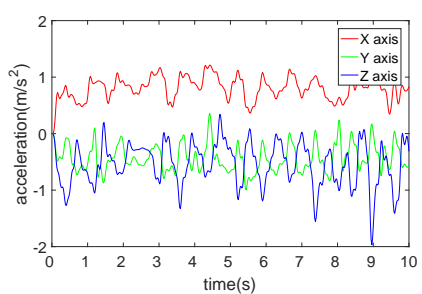

(f)

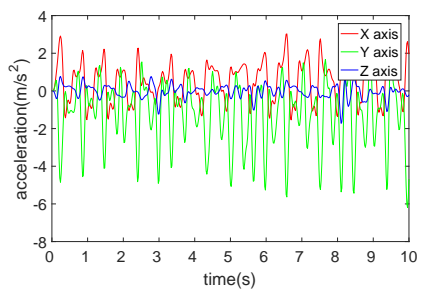

(i)

Figure 4: Wrist acceleration for the nine studied activities: (a) Book Carrying; (b) Door Opening; (c) Floor Mopping; (d) PC Using; (e) Jogging; (f) Standing; (g) Tooth Brushing; (h) Walking; (i) Window Cleaning

to separately conduct the "Door Opening" and "Book Carrying" activities, which are distinct actions, and collected the data for each action. For the other activities, the users performed the corresponding actions continuously for 5-10 minutes. We recorded all sensory data and equally segmented the data offline into 10-seconds samples.

\subsection{Feature extraction}

From the collected data for each segmented activity, we extracted significant motion features to recognize each activity. Specifically, we considered features based on statistics, correlation, frequency, and magnitude. The following features, derived from the inertial data, were taken into accounts:

(1) The statistic features comprised the mean, median, mode, range, skewness, kurtosis, 4-th and 5-th central moment, standard deviation, variance, mean absolute deviation, and sum of absolute values. The description of these features are as follows:

- Mean $[1,6,10,26,34,36]$ : the average of the data segmentation $X$

$$
\mu=\frac{1}{n} \sum_{i=1}^{n} x_{i}
$$




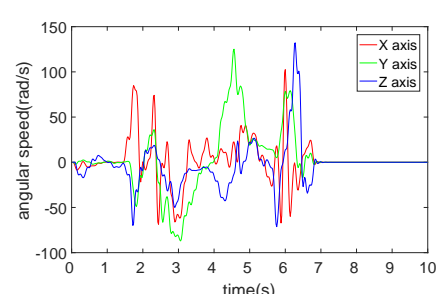

(a)

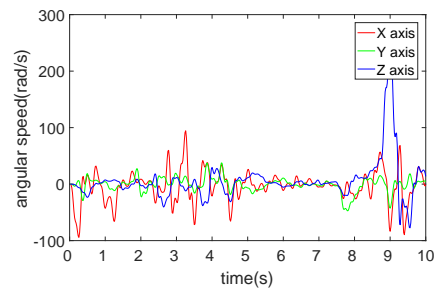

(d)

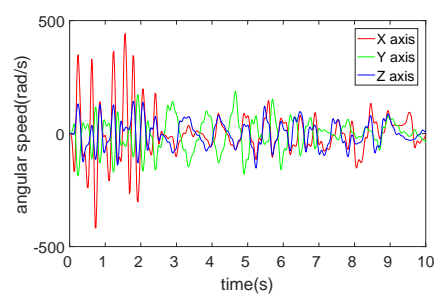

(g)

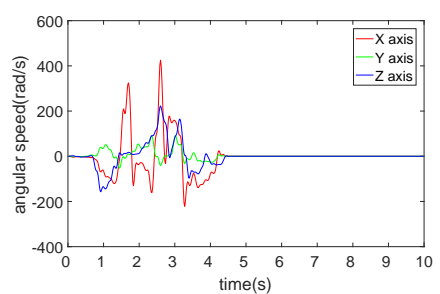

(b)

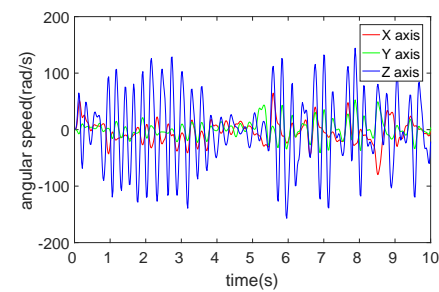

(e)

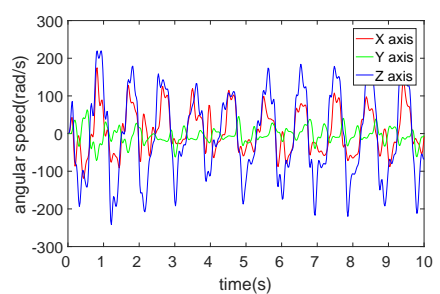

(h)

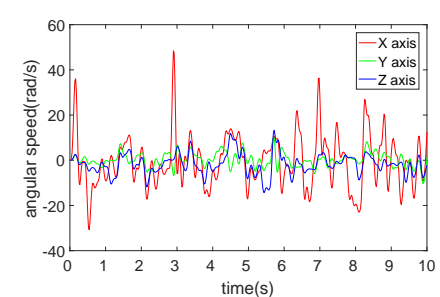

(c)

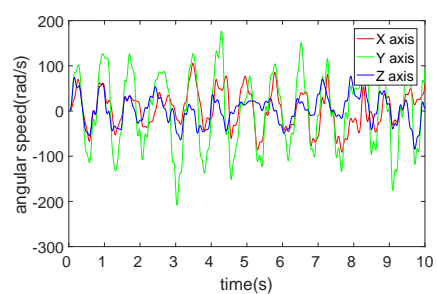

(f)

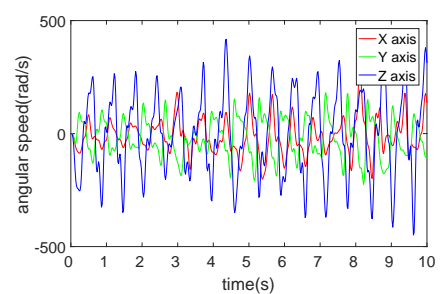

(i)

Figure 5: Wrist angular speeds for the nine studied activities: (a) Book Carrying; (b) Door Opening; (c) Floor Mopping; (d) PC Using; (e) Jogging; (f) Standing; (g) Tooth Brushing; (h) Walking; (i) Window Cleaning

- Median $[1,7,26]$ : the middle value of the data segmentation $X$

$$
\tilde{\mu}=\left\{\begin{array}{cc}
X\left(\frac{n+1}{2}\right) & n: \text { oddvalue } \\
\frac{X\left(\frac{n}{2}\right)+X\left(\frac{n}{2}+1\right)}{2} & n: \text { evenvalue }
\end{array}\right.
$$

- Mode $[1,26]$ : the most frequently occurring value $\hat{\mu}$ in the data segment $X$ - Range [1]: the maximum difference between the values within the sliding window

$$
r=\max _{i=1}^{n}\left(x_{i}\right)-\min _{i=1}^{n}\left(x_{i}\right)
$$

- Skewness [1,26]:

$$
\gamma_{1}=\frac{\frac{1}{n} \sum_{i=1}^{n}\left(x_{i}-\bar{x}\right)^{3}}{\left(\sqrt{\frac{1}{n} \sum_{i=1}^{n}\left(x_{i}-\bar{x}\right)^{2}}\right)^{3}}
$$

- Kurtosis $[1,26]$ :

$$
\gamma_{2}=\frac{\frac{1}{n} \sum_{i=1}^{n}\left(x_{i}-\bar{x}\right)^{4}}{\left(\sqrt{\frac{1}{n} \sum_{i=1}^{n}\left(x_{i}-\bar{x}\right)^{2}}\right)^{2}}
$$

- Moment $[1,10,34,36]$ : we selected the 4 -th and 5 -th moments of the data segment as derived 


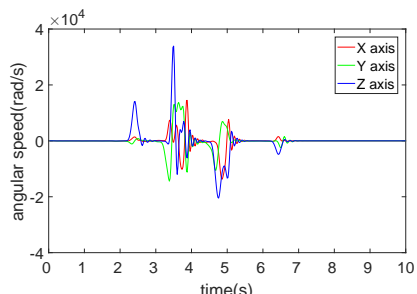

(a)

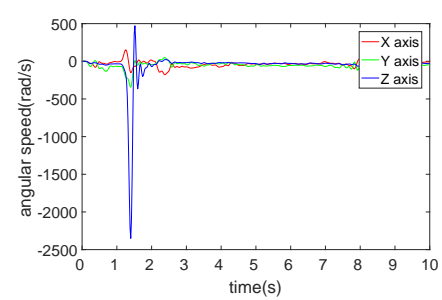

(d)

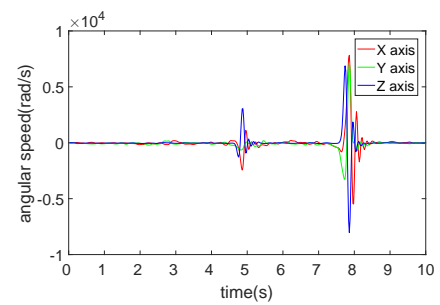

(g)

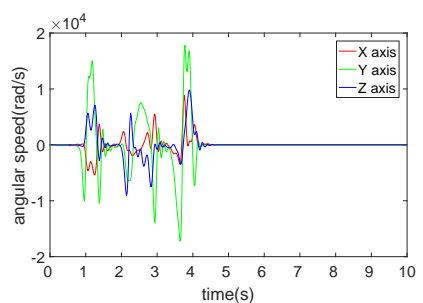

(b)

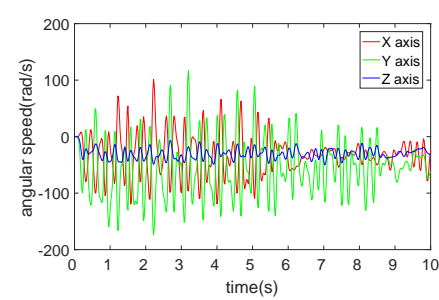

(e)

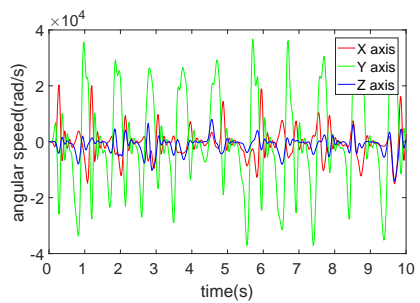

(h)

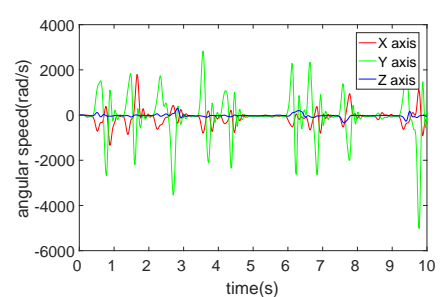

(c)

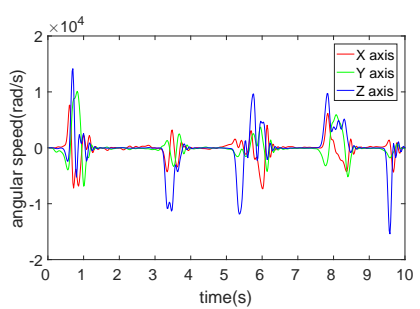

(f)

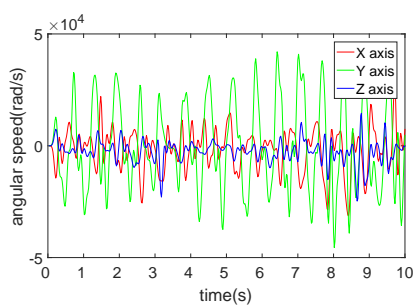

(i)

Figure 6: Angular speeds measured at the(g) Tooth Brushing; (h) Walking; (i) Window Cleaning features.

$$
\begin{aligned}
& \mu_{4}=\frac{1}{n} \sum_{i=1}^{n}\left(x_{i}-\bar{x}\right)^{4} \\
& \mu_{5}=\frac{1}{n} \sum_{i=1}^{n}\left(x_{i}-\bar{x}\right)^{5}
\end{aligned}
$$

- Standard deviation $[6,7,10,34,36]$ :

$$
\sigma=\sqrt{\frac{1}{n} \sum_{i=1}^{n}\left(x_{i}-\mu\right)^{2}}
$$

- Variance $[1,6,26]$ :

$$
\sigma^{2}=\frac{1}{n} \sum_{i=1}^{n}\left(x_{i}-\mu\right)^{2}
$$

(2) Magnitude-based features comprised the mean absolute deviation (MAD), signal magnitude area (SMA), root mean square (RMS), intensity of movement (IM), sum of absolute values (SAV). The description of these features are as follows:

- Mean absolute deviation [6,10]:

$$
M A D=\frac{1}{n} \sum_{i=1}^{n}\left|x_{i}-\mu\right|
$$


- Signal Magnitude Area [7, 10,34,36]:

$$
S M A=\frac{1}{n} \sum_{i=1}^{n}\left|x_{i}-\bar{x}\right|
$$

- Root mean square $[6,10]$ :

$$
R M S=\sqrt{\frac{1}{n} \sum_{i=1}^{n} x_{i}^{2}}
$$

- Intensity of movement $[10,11]$ :

$$
I M=\frac{1}{n} \sum_{i=1}^{n}\left|x_{n-i+1}-x_{n-i}\right|
$$

- Sum of absolute values [10]:

$$
S A=\frac{1}{n} \sum_{i=1}^{n}\left|x_{i}-\bar{x}\right|
$$

(3) Correlation-based features comprised the correlation-between-axes values. The description of these features are as follows:

- Correlation between axes $[6,10,36]$ : the Pearson correlation coefficient between two data segments $X=x_{1}, x_{2}, \ldots, x_{n}$ and $Y=y_{1}, y_{2}, \ldots, y_{n}$ is defined as:

$$
\rho_{X Y}=\frac{n\left(\sum_{i=1}^{n} x_{i} y_{i}\right)-\sum_{i=1}^{n} x_{i} \sum_{i=1}^{n} y_{i}}{\left(n \sum_{i=1}^{n} x_{i}^{2}-\left(\sum_{i=1}^{n} x_{i}\right)^{2}\right)\left(n \sum_{i=1}^{n} y_{i}^{2}-\left(\sum_{i=1}^{n} y_{i}\right)^{2}\right)}
$$

(4) Frequency-domain features comprised the average energy (AE), dominant frequency (DF), and amplitude values. The description of these features are as follows:

- Average energy $[1,3,6,10,34,36]$ : the average of the energies in three axes of the triaxial sensors. The energy is calculated by summing all squared discrete fast Fourier transform (FFT) component magnitudes of the signal.

- Dominant frequency $[7,26]$ : the frequency determined in the FFT-domain at which the discrete FFT component magnitude is the largest.

- Amplitude $[1,7,26]$ : the amplitude at the dominant frequency in the FFT-domain.

To extract the indicators from the plantar-pressure data obtained from the eight pressure sensors, we derived the following features:

- Statistical features [38]: these features comprised the mean, max, and standard deviation of the pressure data. The formulas for these functions are the same as those described above. - Correlation between the counterpart sensors from both feet [38]: we calculated the correlation of the plantar-pressure data collected from counterpart sensors that are placed correspondingly under two feet. The correlation computation is as described in Eq. 15.

- Pressure area [13]: this feature is computed as the integral of the pressure data. In an earlier study [13], we used this feature for each step to classify three ambulatory activities with an accuracy rate of $95.2 \%$. The formula to calculate this feature for a segment of $k$-th pressure, $P_{k}=\left\{P_{k}(1), P_{k}(2),, \ldots, P_{k}(n)\right\}$, is described as follows:

$$
S_{P_{k}}=\sum_{i=1}^{n} P_{k}(i)
$$


We applied different approaches to the acceleration, angular speed and plantar-pressure data to obtain features for classification. Table 2 summarizes the list of derived features. Here, $(x, y, z)$ and $(b, r s, l s)$ represent the $(X, Y, Z)$ axes and (band, right shoe, left shoe), respectively. The second column of the table denotes the notations of the corresponding feature names in the first column. The third column contains the listed segmented data that are employed to extract the corresponding feature groups in the first column. The last four rows, i.e., mean, max, standard deviation, and pressure area, are the four features that are extracted from the plantar-pressure data.

Table 2: List of derived features.

\begin{tabular}{|c|c|c|}
\hline Feature Name & Notation & Applied Data \\
\hline mean & $\mu$ & \multirow{10}{*}{$a_{x}^{b}, a_{y}^{b}, a_{z}^{b}, \omega_{x}^{b}, \omega_{y}^{b}, \omega_{z}^{b}, a_{x}^{r s}, a_{y}^{r s}, a_{z}^{r s}, \omega_{x}^{r s}, \omega_{y}^{r s}, \omega_{z}^{r s}, a_{x}^{l s}, a_{y}^{l s}, a_{z}^{l s}, \omega_{x}^{l s}, \omega_{y}^{l s}, \omega_{z}^{l s}$} \\
\hline mode & $\hat{\mu}$ & \\
\hline median & $\tilde{\mu}$ & \\
\hline range & $r$ & \\
\hline skewness & $\gamma_{1}$ & \\
\hline kurtosis & $\gamma_{2}$ & \\
\hline 4-th moment & $\mu_{4}$ & \\
\hline 5-th moment & $\mu_{5}$ & \\
\hline standard deviation & $\sigma$ & \\
\hline variance & $\sigma^{2}$ & \\
\hline mean absolute deviation & $M A D$ & \multirow{5}{*}{$a_{x}^{b}, a_{y}^{b}, a_{z}^{b}, \omega_{x}^{b}, \omega_{y}^{b}, \omega_{z}^{b}, a_{x}^{r s}, a_{y}^{r s}, a_{z}^{r s}, \omega_{x}^{r s}, \omega_{y}^{r s}, \omega_{z}^{r s}, a_{x}^{l s}, a_{y}^{l s}, a_{z}^{l s}, \omega_{x}^{l s}, \omega_{y}^{l s}, \omega_{z}^{l s}$} \\
\hline signal magnitude area & $S M A$ & \\
\hline root mean square & $R M S$ & \\
\hline intensity of movement & $I M$ & \\
\hline sum of absolute values & $S A$ & \\
\hline correlation & $\rho$ & $\begin{array}{c}\left(a_{x}^{b}, a_{y}^{b}\right),\left(a_{y}^{b}, a_{z}^{b}\right),\left(a_{z}^{b}, a_{x}^{b}\right),\left(\omega_{x}^{b}, \omega_{y}^{b}\right),\left(\omega_{y}^{b}, \omega_{z}^{b}\right),\left(\omega_{z}^{b}, \omega_{x}^{b}\right), \\
\left(a_{x}^{r s}, a_{y}^{r s}\right),\left(a_{y}^{r s}, a_{z}^{r s}\right),\left(a_{z}^{r s}, a_{x}^{r s}\right),\left(\omega_{x}^{r s}, \omega_{y}^{r s}\right),\left(\omega_{y}^{r s}, \omega_{z}^{r s}\right),\left(\omega_{z}^{r s}, \omega_{x}^{r s}\right), \\
\left(a_{x}^{l s}, a_{y}^{l s}\right),\left(a_{y}^{l s}, a_{z}^{l s}\right),\left(a_{z}^{l s}, a_{x}^{l s}\right),\left(\omega_{x}^{l s}, \omega_{y}^{l s}\right),\left(\omega_{y}^{l s}, \omega_{z}^{l s}\right),\left(\omega_{z}^{l s}, \omega_{x}^{l s}\right)\end{array}$ \\
\hline average energy & $A E$ & $\begin{array}{c}\left(a_{x}^{b}, a_{y}^{b}, a_{z}^{b}\right),\left(\omega_{x}^{b}, \omega_{y}^{b}, \omega_{z}^{b}\right) \\
\left(a_{x}^{r s}, a_{y}^{r s}, a_{z}^{r s}\right),\left(\omega_{x}^{r s}, \omega_{y}^{r s}, \omega_{z}^{r s}\right) \\
\left(a_{x}^{l s}, a_{y}^{l s}, a_{z}^{l s}\right),\left(\omega_{x}^{l s}, \omega_{y}^{l s}, \omega_{z}^{l s}\right)\end{array}$ \\
\hline dominant frequency & $f_{d}$ & $a_{x}^{b}, a_{y}^{b}, a_{z}^{b}, \omega_{x}^{b}, \omega_{y}^{b}, \omega_{z}^{b}, a_{x}^{r s}, a_{y}^{r s}, a_{z}^{r s}, \omega_{x}^{r s}, \omega_{y}^{r s}, \omega_{z}^{r s}, a_{x}^{l s}, a_{y}^{l s}, a_{z}^{l s}, \omega_{x}^{l s}, \omega_{y}^{l s}, \omega_{z}^{l s}$ \\
\hline amplitude & $A_{f d}$ & $a_{x}^{b}, a_{y}^{b}, a_{z}^{b}, \omega_{x}^{b}, \omega_{y}^{b}, \omega_{z}^{b}, a_{x}^{r s}, a_{y}^{r s}, a_{z}^{r s}, \omega_{x}^{r s}, \omega_{y}^{r s}, \omega_{z}^{r s}, a_{x}^{l s}, a_{y}^{l s}, a_{z}^{l s}, \omega_{x}^{l s}, \omega_{y}^{l s}, \omega_{z}^{l s}$ \\
\hline mean & $\mu$ & \\
\hline $\max$ & $\max$ & $P_{1}^{r s}, P_{2}^{r s}, P_{3}^{r s}, P_{4}^{r s}, P_{5}^{r s}, P_{6}^{r s}, P_{7}^{r s}, P_{8}^{r s}, P_{1}^{l s}, P_{2}^{l s}, P_{3}^{l s}, P_{4}^{l s}, P_{5}^{l s}, P_{6}^{l s}, P_{7}^{l s}, P_{8}^{l s}$ \\
\hline standard deviation & $\sigma$ & \\
\hline pressure area & $S$ & $P_{1}^{r s}, P_{2}^{r s}, P_{3}^{r s}, P_{4}^{r s}, P_{5}^{r s}, P_{6}^{r s}, P_{7}^{r s}, P_{8}^{r s}, P_{1}^{l s}, P_{2}^{l s}, P_{3}^{l s}, P_{4}^{l s}, P_{5}^{l s}, P_{6}^{l s}, P_{7}^{l s}, P_{8}^{l s}$ \\
\hline
\end{tabular}

\subsection{Feature selection}

In this paper, we used the filter technique to reduce the computational complexity and accelerate feature selection. Specifically, we applied the RELIEF-F method [15] to score the extracted features. The RELIEF-F method is based on the average of the $\mathrm{K}$ near misses from each class. We analyzed and ranked all features derived from the sensory data, and then, selected subsets of important features for classification. Here, sensory data from each device are independently passed into the feature extractor to derive the features. The feature selector scores and ranks these features to form an effective subset of feature for classification. We also merged the corresponding features from the devices to assess activity recognition with sensor fusion schemes. 


\subsection{Classification}

To classify nine daily living activities, we constructed two classifiers, i.e., KNN and CART. Using the results from the feature selector, we passed the selected features subset into the classifiers and compared the performance of the predictive models. Given a feature subset of $m$ training samples $\left\{x^{(i)}, y^{(i)}\right\}_{i=1}^{m}$, the KNN classifier classifies each test sample $x^{(k)}$ based on the Euclidean distance of the test sample $x^{(k)}$ to each training sample $x^{(i)}$. The Euclidean distance is calculated as follows:

$$
d\left(x^{(k)}, x^{(i)}\right)=\sqrt{\sum_{j=1}^{N}\left(x_{j}^{(i)}-x_{j}^{(k)}\right)^{2}} .
$$

The $k$ training samples corresponding to the $k$ shortest distances are used to categorize the test sample. We utilize the bagging approach for KNN classification. Specifically, the $k$ training samples that have the shortest distances to the test sample are bagged in a group $G$. Then, the labels of these $k$ samples are considered. The test sample is classified into the class that has the highest occurrence probability in the group $G$. The decision tree classifier is constructed based on a flowchart-like structure. At each internal node, a value test is conducted on an attribute of the selected feature subset to split the outcome into two groups. In other words, at each node, the tree divides the data into two branches. This splitting process is recursively repeated until either the groups at a node belong to the same class or the groups have the same attribute values.

\section{Experimental results}

In the experiments, we set the sampling rate of the system to $50 \mathrm{~Hz}$ and required two participants to the wear smart band and smart shoes and perform each segmented activity for 10 seconds to collect data. We used a separate sliding window to divide the data series into equal segments of recorded data. Each segment was used as a sample of an activity for recognition. In total, there were 363 samples of nine activities. We applied the proposed method presented to each sample to recognize its corresponding activity label. First, we removed noise in the signal by applying a low-pass Butterworth filter with the order of $N=6$ and cut-off frequency $f_{c}=10 \mathrm{~Hz}$. Then, we create feature sets using a feature extractor with the operations listed in Table 2. Finally, applying the feature selection scheme RELIEF-F with $\mathrm{K}=10$ on the feature sets, we identified the most important features for classifying activities on each device and fusion of devices.

Figure $7 \mathrm{a}$ and $7 \mathrm{~b}$ depict results of feature selection for the features derived at the right smartshoe and the corporation of both shoes in the descending order of their importance, respectively. Figure $7 \mathrm{c}$ depicts the 20 most important features derived at the band using the feature selection scheme in the descending order of their importance. Fusing all features created from all sensors of all devices and evaluating their importance, we obtain a set of 20 most important features as shown in Figure 7d. From this evaluation, we can see that the features derived from inertial data at the foot contribute a more important impact on recognition of the 9 activities than the features derived from inertial data at the wrist because the first 20 most important features in Figure $7 \mathrm{~b}$ and $7 \mathrm{a}$ are all selected from data collected on the smart-shoes. Furthermore, it can be observed from Figure $7 \mathrm{~b}$ and $7 \mathrm{a}$ that the two smart-shoes have a similarly influence on the recognition. From Figure 7a, $7 \mathrm{~b}$ and $7 \mathrm{c}$, we can draw a statement that using gyroscope sensors in the smart-shoes provides a better performance of recognizing the nine activities than using accelerometers at the feet because the all 20 first features in these figures are related to the smartshoes. From the feature subsets, we applied 2 classifiers, i.e., KNN and CART, to recognize the activity of each sample. We examined the prediction on various feature sets to evaluate the 

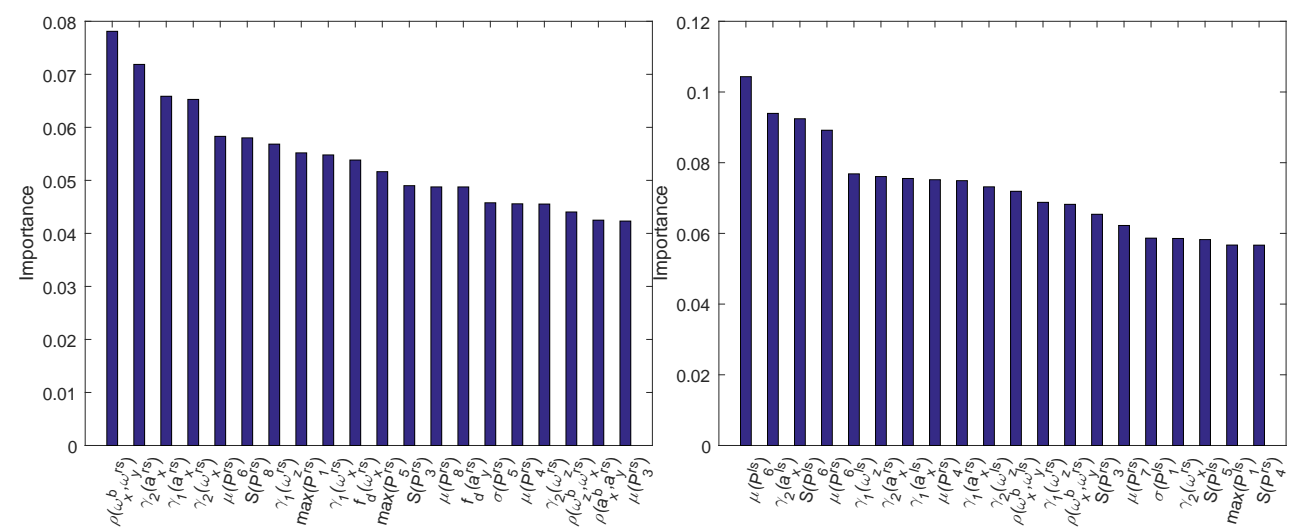

(a)

(b)
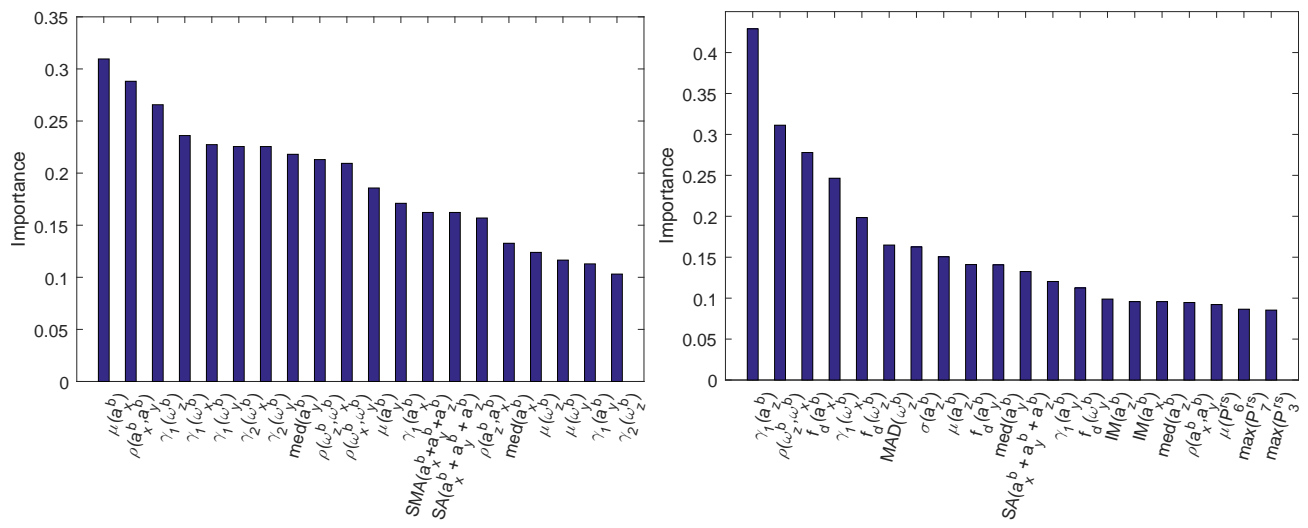

(c)

(d)

Figure 7: List of most important features for all activity classification: (a) Important features of a smart shoe; (b) Important features of two smart shoes; (c) Important features of smart band; (d) Important features of the sensor fusion

ability of each classifier. It should be noted that we do not need to test all features in the feature sets. Because the importance values of the features in each set have been measured in FS step, we sequentially selected features from the evaluated set according to descending importance. Herein, we examined sets of 1-40 features for each case. Figure 8 shows the accuracy rates of different classifiers with respect to the number of features for each case.

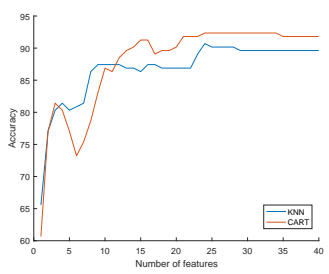

(a)

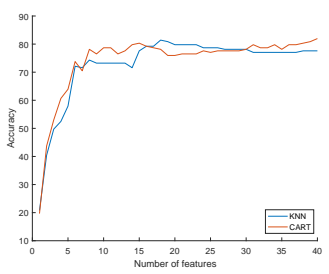

(b)

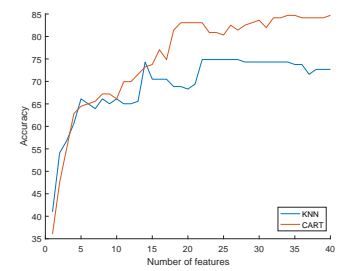

(c)

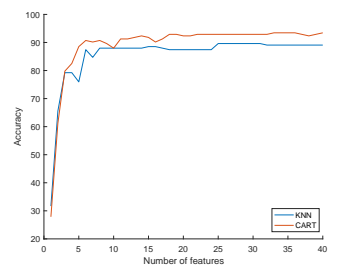

(d)

Figure 8: Accuracy of all activity classification versus the number of selected features: (a) A smart band; (b) A smart shoe; (c) A pair of smart shoes; (d) Sensor fusion

It can be seen that in overall, the KNN classifier exhibits a better performance than the CART classifier for recognizing the nine studied activities. Using the KNN classifier, the maximal 
result $(89.1 \%)$ for recognition is obtained with 21 features for the smart band case. For features derived from a smart shoe, the KNN-based recognition exhibits the optimal performance at 39 features. More features are required for the best recognition when using the smart shoe than when using the smart band, but the result is much lower $(77 \%)$. Merging two smart shoes can improve the recognition up to $80 \%$ with 33 features. Finally, the data fusion scheme provides the best result $(90.7 \%)$ with the fewest features used, i.e., 18 features. Therefore, we recommend the use of the data fusion scheme and application of the KNN classifier to recognize the nine studied activities with the 18 features depicted in Figure $7 \mathrm{~d}$.

Table 3 shows the details of the recognition results for all nine activities. The smart band was effective in recognizing the Door Opening and PC Using activities, which are significantly dependent on the hand movement. Particularly, the precision, recall and f1-score values of the recognition for Door Opening and PC Using using the smart band's sensors were all higher than the recognition values using the total combination of sensors. The f1-scores when using the smart band's sensors were $3.6 \%$ and $2.1 \%$ higher than the f1-scores when using the total combination of sensors for Door Opening and PC Using, respectively. The smart shoes can recognize the Walking and Jogging activities better than the smart band. The shoes-based recognition of these activities yielded an f1-score improvement of $2.2 \%$ and $2.0 \%$ compared with that of the smart band-based recognition. For the remain activities, the smart band-based recognition clearly surpasses the smart-shoes-based recognition. Combining all sensors provided the best recognition ability for all nine activities. Using the sensor fusion approach, the f1-score of all activities was $1.6 \%$ and $11.3 \%$ better than when using the smart-band and smart-shoe approaches, respectively.

Table 3: Classification metrics of each activity with different approaches

\begin{tabular}{|l|c|c|c|c|c|c|c|c|c|}
\hline Sensing method & \multicolumn{3}{|c|}{ Smart-band sensors } & \multicolumn{3}{c|}{ Smart-shoe sensors } & \multicolumn{3}{c|}{ Combination of sensors } \\
\hline Activity & precision & recall & f1-score & precision & recall & f1-score & precision & recall & f1-score \\
\hline BookLoading & $88.2 \%$ & $93.8 \%$ & $90.9 \%$ & $73.3 \%$ & $68.8 \%$ & $71.0 \%$ & $88.2 \%$ & $93.8 \%$ & $90.9 \%$ \\
\hline DoorOpening & $87.5 \%$ & $93.3 \%$ & $90.3 \%$ & $68.4 \%$ & $86.7 \%$ & $76.5 \%$ & $86.7 \%$ & $86.7 \%$ & $86.7 \%$ \\
\hline PCUsing & $100.0 \%$ & $95.7 \%$ & $97.8 \%$ & $95.7 \%$ & $95.7 \%$ & $95.7 \%$ & $95.7 \%$ & $95.7 \%$ & $95.7 \%$ \\
\hline Standing & $94.1 \%$ & $80.0 \%$ & $86.5 \%$ & $71.4 \%$ & $75.0 \%$ & $73.2 \%$ & $94.1 \%$ & $80.0 \%$ & $86.5 \%$ \\
\hline ToothBrushing & $89.3 \%$ & $96.2 \%$ & $92.6 \%$ & $83.3 \%$ & $57.7 \%$ & $68.2 \%$ & $92.6 \%$ & $96.2 \%$ & $94.3 \%$ \\
\hline Mopping & $95.8 \%$ & $95.8 \%$ & $95.8 \%$ & $82.1 \%$ & $95.8 \%$ & $88.5 \%$ & $96.0 \%$ & $100.0 \%$ & $98.0 \%$ \\
\hline WindowsCleaning & $90.9 \%$ & $87.0 \%$ & $88.9 \%$ & $77.3 \%$ & $73.9 \%$ & $75.6 \%$ & $95.7 \%$ & $95.7 \%$ & $95.7 \%$ \\
\hline Walking & $86.4 \%$ & $95.0 \%$ & $90.5 \%$ & $90.5 \%$ & $95.0 \%$ & $92.7 \%$ & $95.0 \%$ & $95.0 \%$ & $95.0 \%$ \\
\hline Jogging & $90.0 \%$ & $93.8 \%$ & $91.8 \%$ & $93.8 \%$ & $93.8 \%$ & $93.8 \%$ & $100.0 \%$ & $93.8 \%$ & $96.8 \%$ \\
\hline Mean & $91.4 \%$ & $92.3 \%$ & $91.7 \%$ & $81.8 \%$ & $82.5 \%$ & $81.7 \%$ & $93.8 \%$ & $93.0 \%$ & $93.0 \%$ \\
\hline
\end{tabular}

Figure 9 shows the average value of the performance metrics of the smart band-smart shoes fusion (B-S Fusion) and the reference methods. The multi-sensor method [9] yielded the best recognition result by fusing data from the accelerometers on the chest waist, thigh, and side; then, extracting the mean and var features; and, finally, using a decision tree classifier to recognize the activities. We utilized this approach on the collected database by applying the presented feature extractor on acceleration data from all devices and use a decision tree on the extracted feature set to evaluate the method performance. The RF $+\mathrm{KNN}$ method [25] extracted 12 features for each sample of acceleration collected at the wrist based on the mean, energy, frequency entropy, correlation between axes. Then, a variety of classifiers was applied to classify activities. Among these classifiers, a combination of random forest (RF) and KNN using the average of probabilities approach provided the best result.

It is clear that the proposed fusion approach outperformed the reference methods for activity recognition in terms of all four metrics, i.e., precision, recall, $\mathrm{f}-1$ score, and accuracy. In particular, the accuracy rate of the B-S fusion method was $7.9 \%$ and $7.1 \%$ higher on average than the 


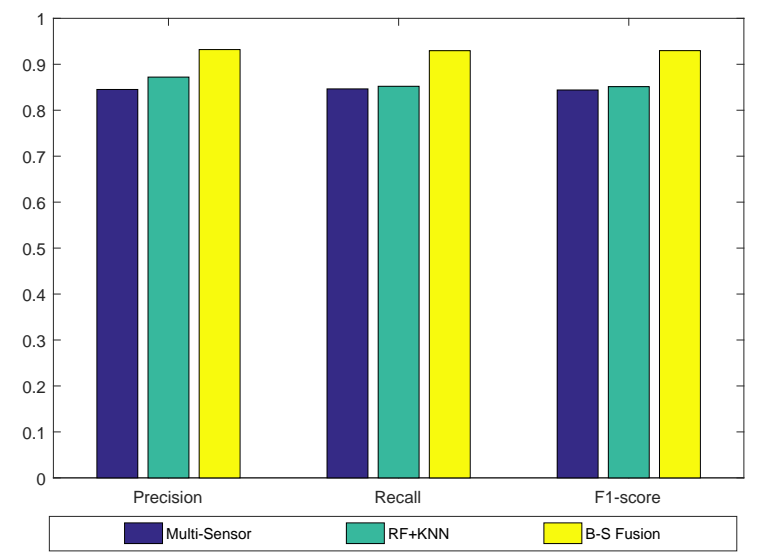

Figure 9: Performance comparison of the proposed method and the reference methods

mMulti-sensor [9] and RF + KNN [25] in accuracy rate, respectively. The B-S fusion method recognized activities better than the RF + KNN method because the B-S fusion method utilized more sensors in different locations, which in turn can better track the motion of different body parts during daily activities. This result confirms once again the results shown in Figure 8 and Table 3. In particular, when applying the same technique, the results of using the B-S fusion data surpassed the results of using only wrist-related data. Although the multi-sensor method also used a combination of accelerometers at the right wrist and both feet, this method did not utilize gyroscope data. Besides, the original paper for the multi-sensor method [9] dealt with four ambulatory activities, i.e., walking, standing, lying, sitting, and transitional states of these activities. Therefore, their selected features were not well-performed in this study.

\section{Conclusion}

This paper addressed the issues of sensor placement, feature selection, and classification in daily activity recognition using wearable sensors. A combination of a smart band and a pair of smart shoes was used to collect motion data from users and transfer data to a smartphone for offline activity classification. In our experiments, the participants wore the smart shoes and smart band and then, completed the experiments separately for each activity. Using a separate sliding window, we divided the data series into various segments of recorded data. From the segmented samples, we derived features and applied a filter feature selection algorithm to select important subsets. Two classifiers, i.e, KNN and CART, were employed to recognize the activities. The experimental results showed that the KNN classifier yielded better results than the CART classifier; the smart band outperformed the smart shoes in recognizing all nine activities in terms of accuracy and fewer features; the smart band is the best fit for recognizing the two hand-movement-dominated activities; the smart shoes are the best choice for the two footmovement-dominated activities; and using a total combination of sensors is the best approach for recognizing all nine daily activities in terms of both accuracy and the number of required features.

\section{Funding}

This research was supported by the Basic Science Research Program through the National Research Foundation of Korea (NRF) funded by the Ministry of Education 
(NRF-2018R1D1A1A09083894). This research was also supported by the National Research Foundation of Korea (NRF) funded by the Korean Government (MSIP) (NRF-2016R1A5A1012966).

\section{Conflict of interest}

The authors declare no conflict of interest.

\section{Bibliography}

[1] Banos, O.; Damas, M.; Pomares, H.; Prieto, A.; Rojas, I. (2012). Daily living activity recognition based on statistical feature quality group selection, Expert Syst. Appl., 39, 8013$8021,2012$.

[2] Banos, O.; Moral-Munoz, J.; Diaz-Reyes, I.; Arroyo-Morales, M.; Damas, M.; HerreraViedma, E.; Hong, C.; Lee, S.; Pomares, H.; Rojas, I.; Villalonga, C. (2015). mDurance: A novel mobile health system to support trunk endurance assessment, Sensors, 15, 13159$13183,2015$.

[3] Bao, L.; Intille, S.S. (2004). Pervasive Computing; Vol. 3001, Lecture Notes in Computer Science, Springer Berlin Heidelberg, 1 - 17, 2004.

[4] Bruno, B.; Mastrogiovanni, F.; Sgorbissa, A. (2015). Wearable inertial sensors: Applications, challenges, and public test benches, IEEE Robot. Autom. Mag., 22, 116-124, 2015.

[5] Bulling, A.; Blanke, U.; Schiele, B. (2014). A tutorial on human activity recognition using body-worn inertial sensors, ACM Comput. Surv., 46, 1-33, 2014.

[6] Chen, Y.P.; Yang, J.Y.; Liou, S.; Lee, G.; Wang, J.S. (2008). Online classifier construction algorithm for human activity detection using a tri-axial accelerometer, Appl. Math. Comput., 205, 849-860, 2008.

[7] Concepcion., A. D.L.; Morillo, S.; Gonzalez, A.; Ramírez, O. (2014). Discrete techniques applied to low-energy mobile human activity recognition. A new approach, Expert Syst. Appl., 41, 6138-6146, 2014.

[8] Dzitac, S ; Vesselenyi, T. ; Popper, L. et al. (2010). Fuzzy Algorithm for Human Drowsiness Detection Devices, Studies in Informatics and Control, 19(4), 419-426, 2010.

[9] Gao, L.; Bourke, A.; Nelson, J. (2014). Evaluation of accelerometer based multi-sensor versus single-sensor activity recognition systems, Med. Eng. Phys., 36, 779-785, 2014.

[10] González, S.; Sedano, J.; Villar, J.R.; Corchado, E.; Herrero, Á.; Baruque, B. (2015). Features and models for human activity recognition, Neurocomputing, 167, 52-60, 2015.

[11] Gyorbiró, N.; Fábián, Á.; Hományi, G. (2008). An activity recognition system for mobile phones, Mob. Networks Appl., 14, 82-91, 2008.

[12] Huynh, T.; Schiele, B. (2007). Towards less supervision in activity recognition from wearable sensors. 10th IEEE Int. Symp. Wearable Comput., 3-10, 2006.

[13] Jeong, G.M.; Truong, P.H.; Choi, S.I. (2017). Classification of three types of walking activities regarding stairs using plantar pressure sensors, IEEE Sens. J., 17, 2638-2639, 2017. 
[14] Jia, Y. (2009). Diatetic and exercise therapy against diabetes mellitus, 2009 Second Int. Conf. Intell. Networks Intell. Syst., 693-696, 2009.

[15] Kononenko, I. (1994). Estimating attributes: Analysis and extensions of RELIEF. Proc. Eur. Conf. Mach. Learn., 171-182, 1994.

[16] Lara, O.; Labrador, M. (2013). A survey on human activity recognition using wearable sensors, IEEE Commun. Surv. Tutorials, 15, 1192-1209, 2013.

[17] Laudanski, A.; Brouwer, B.; Li, Q. (2015). Activity classification in persons with stroke based on frequency features, Med. Eng. Phys., 37, 180-186, 2015.

[18] Liu, Y.; Nie, L.; Liu, L.; Rosenblum, D. (2016). From action to activity: Sensor-based activity recognition, Neurocomputing, 181, 108-115, 2016.

[19] Lowe, S.; ÓLaighin, G. (2014). Monitoring human health behaviour in one's living environment: A technological review, Med. Eng. Phys., 36, 147-168, 2014.

[20] Mannini, A.; Sabatini, A.M. (2010). Machine learning methods for classifying human physical activity from on-body accelerometers, Sensors, 10, 1154-1175, 2010.

[21] Mocanu, I.; Scarlat, G.; Rusu, L.; Pandelica, I.; Cramariuc, B. (2018). Indoor Localisation through Probabilistic Ontologies, International Journal of Computers Communications \& Control, 13(6), 988-1006, 2018.

[22] Mukhopadhyay, S.C. (2015). Wearable sensors for human activity monitoring: A review, IEEE Sens. J., 15, 1321-1330, 2015.

[23] Najafi, B.; Aminian, K.; Paraschiv-Ionescu, A.; Loew, F.; Büla, C.J.; Robert, P. (2003). Ambulatory system for human motion analysis using a kinematic sensor: Monitoring of daily physical activity in the elderly, IEEE Trans. Biomed. Eng., 50, 711-723, 2003.

[24] Nguyen, L.; Zeng, M.; Tague, P.; Zhang, J. (2015). Recognizing new activities with limited training data, Proc. 2015 ACM Int. Symp. Wearable Comput. - ISWC '15, ACM Press: New York, USA, 67-74, 2015.

[25] Nguyen, M.; Fan, L.; Shahabi, C. (2015). Activity Recognition Using Wrist-Worn Sensors for Human Performance Evaluation, 2015 IEEE Int. Conf. Data Min. Work., 164-169, 2015.

[26] Pei, L.; Guinness, R.; Chen, R.; Liu, J.; Kuusniemi, H.; Chen, Y.; Chen, L.; Kaistinen, J. (2013). Human behavior cognition using smartphone sensors, Sensors, 13, 1402-1424, 2013.

[27] Reyes-Ortiz, J.L.; Oneto, L.; Samà, A.; Parra, X.; Anguita, D. (2016). Transition-aware human activity recognition using smartphones, Neurocomputing, 171, 754-767, 2016.

[28] Rodgers, M.; Pai, V.; Conroy, R. (2015). Recent advances in wearable sensors for health monitoring, IEEE Sens. J., 15, 3119-3126, 2015.

[29] Ronao, C.A.; Cho, S.b. (2016). Human activity recognition with smartphone sensors using deep learning neural networks, Expert Syst. Appl., 59, 235-244, 2016.

[30] San-Segundo, R.; Lorenzo-Trueba, J.; Martínez-González, B.; Pardo, J. (2016). Segmenting human activities based on HMMs using smartphone inertial sensors, Pervasive Mob. Comput., 30, 84-96, 2016. 
[31] Sazonov, E.S.; Fulk, G.; Hill, J.; Schutz, Y.; Browning, R. (2011). Monitoring of posture allocations and activities by a shoe-based wearable sensor, IEEE Trans. Biomed. Eng., 58, 983-990, 2011.

[32] Storm, F.; Heller, B.; Mazzà, C. (2015). Step detection and activity recognition accuracy of seven physical activity monitors, PLoS One, 10, e0118723, 2015.

[33] Sung, M.; Marci, C.; Pentland, A. (2005). Wearable feedback systems for rehabilitation, J. Neuroeng. Rehabil., 2, 17, 2005.

[34] Suto, J.; Oniga, S. and Sitar, P.P. (2017). Feature analysis to human activity recognition, Int. J. of Computers Communications \& Control, 12(1), 116-130, 2017.

[35] Wang, J.; Chen, R.; Sun, X.; She, M.; Wu, Y. (2011). Recognizing human daily activities from accelerometer signal, Procedia Eng., 15, 1780-1786, 2011.

[36] Yang, J.Y.; Wang, J.S.; Chen, Y.P. (2008). Using acceleration measurements for activity recognition: An effective learning algorithm for constructing neural classifiers, Pattern Recognit. Lett., 29, 2213-2220, 2008.

[37] Zhang, W.; Thurow, K. and Stoll, R.. (2016). A context-aware mhealth system for online physiological monitoring in remote healthcare. Int. J. Computers Communications 86 Control, 11(1), 142-156, 2016.

[38] Zelun Zhang.; Poslad, S. (2014). Improved use of foot force sensors and mobile phone GPS for mobility activity recognition, IEEE Sens. J., 14, 4340-4347, 2014. 